\title{
DUAL FUNCTORS AND THE RADON-NIKODYM PROPERTY IN THE CATEGORY OF BANACH SPACES
}

\author{
JOAN WICK PELLETIER \\ (Received 28 September 1977; revised 25 May 1978) \\ Communicated by R. H. Street
}

\begin{abstract}
The notion of duality of functors is used to study and characterize spaces satisfying the RadonNikodym property. A theorem of equivalences concerning the Radon-Nikodym property is proved by categorical means; the classical Dunford-Pettis theorem is then deduced using an adjointness argument. The functorial properties of integral operators, compact operators, and weakly compact operators are discussed. It is shown that as an instance of Kan extension the weakly compact operators can be expressed as a certain direct limit of ordinary hom functors. Characterizations of spaces satisfying the Radon-Nikodym property are then given in terms of the agreement of dual functors of the functors mentioned above.
\end{abstract}

Subject classification (Amer. Math. Soc. (MOS) 1970): 46 M 15.

\section{Introduction}

In a previous paper written with Carl Herz (Herz and Pelletier (1976)), we studied dual functors on the category $\mathfrak{B}$ of real Banach spaces and norm-decreasing linear transformations and developed a theory based on the notion we called "computability'. This theory was applied to the class of integral operators, which, though not themselves computable, arise as the dual functor of a computable functor. Intimately bound up with integral operators is the Radon-Nikodym property, whose proper study draws on compact and weakly compact operators.

In the present paper we study these operators and the Radon-Nikodym property from the categorical point of view adopted in Herz and Pelletier (1976). In particular, we characterize which spaces satisfy the Radon-Nikodym property in terms of the behaviour of certain dual functors (Sections 2 and 4). Moreover, we generalize the 
categorical setting - the existence of a left Kan extension-which led in the previous paper to the definition of computability. This makes it possible to describe the weakly compact operators as the left Kan extension of the hom functor on a certain subcategory of $\mathfrak{B}$ (Section 3 ), much as the compact operators were described in Herz and Pelletier (1976), 1.3 and 3.2.

The essential approach here is that we exploit the behaviour of several key functors on 'manageable' subcategories of $\mathfrak{B}$, in particular on $\mathfrak{F B}$ and $\mathfrak{R B}$, the full subcategories of all finite dimensional and reflexive Banach spaces, respectively. In many cases, functors are completely determined by their behaviour, say, on $\mathfrak{F}^{\mathfrak{B}}$ (that is they are $\mathfrak{F}$-computable). In others, the relationships between functors and their ' $F$-computable parts' are significant. It is in this light that we present our Theorems 4.1 and 4.2 , which state that the Radon-Nikodym property is connected to the agreement of various functors with their $\mathfrak{Z}$ - or $\mathfrak{R}$-computable parts.

Before presenting the categorical set-up alluded to above, let us first recall the basic definitions and notations used in the study of functors and the duality of functors on $\mathfrak{B}$.

We let $\operatorname{HOM}(X, Y)$ denote the Banach space whose unit ball is $\mathfrak{B}(X, Y)$, the set of all norm-decreasing linear transformations from $X$ to $Y$. All functors are assumed to be strong endofunctors on $\mathfrak{B}$, that is functors $F$ such that the induced map $\operatorname{HOM}(X, Y) \rightarrow \operatorname{HOM}(F X, F Y)$ is a morphism in $\mathfrak{B}$. A $\mathfrak{B}$-natural transformation $t: F \rightarrow G$ is a family $\left\{t_{X} \in \mathfrak{B}(F X, G X)\right\}$ satisfying the property that for any $f \in \mathfrak{B}(X, Y), G f^{\circ} t_{X}=t_{Y} \circ F f$. We let $\mathfrak{B}(F, G)$ denote the set of $\mathfrak{B}$-natural transformations from $F$ to $G$ and $N A T(F, G)$ denote the Banach space whose unit ball is $\mathfrak{B}(F, G)$ with norm given by $\|t\|=\sup \left\{\left\|t_{X}\right\|: X \in \mathfrak{B}\right\}$. (One can refer to Mityagin and Švarc (1964) for a proof that $\|t\|<\infty$.)

The functor $D: \mathfrak{B}^{\mathfrak{B}} \rightarrow\left(\mathfrak{B}^{\mathfrak{B}}\right)^{\text {op }}$ is defined as follows: for $X \in \mathfrak{B}, f \in \mathfrak{B}(X, Y)$, we have

$$
\begin{gathered}
D F X=N A T\left(F, \Sigma_{\mathrm{x}}\right), \\
D F(f)(\gamma)=\Sigma_{f} \circ \gamma \quad \text { for } \gamma \in D F X,
\end{gathered}
$$

where $\Sigma_{X} Y=X \otimes Y$ denotes the Banach space tensor product (the algebraic tensor product completed with respect to the greatest cross norm). $D F$ is called the dual functor of $F$.

We consider the restriction functor

$$
U: \mathfrak{B}^{\mathfrak{B}} \rightarrow \mathfrak{B}^{\mathfrak{A}},
$$

where $\mathfrak{A}$ is a full subcategory of $\mathfrak{B}$, and $\mathfrak{B}^{(-)}$denotes the category of (strong) functors from $(-)$ to $\mathfrak{B}$. If $I$ denotes the inclusion functor from $\mathfrak{A}$ to $\mathfrak{B}$, then the left adjoint of $U, \operatorname{Lan}_{1}: \mathfrak{B}^{\mathfrak{P}} \rightarrow \mathfrak{B}^{\mathfrak{P}}$, is called the (enriched) left Kan extension along $I$. 
Specifically, if $F \in \mathfrak{B}^{\mathfrak{A}}, G \in \mathfrak{B}^{\mathfrak{P}}$, then we have the commutative diagram

and the isomorphism

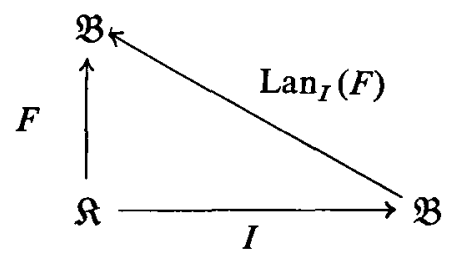

$$
N A T_{\mathfrak{B}^{\mathbb{B}}}\left(\operatorname{Lan}_{I}(F), G\right) \cong N A T_{\mathfrak{B}^{\mathcal{A}}}(F, G \circ I) .
$$

The theory of ordinary Kan extensions is discussed at length in MacLane (1971) and conditions are given for their existence in terms of both colimits and coends.

The situation for enriched Kan extensions is more complicated and requires the approach via coends. This was first introduced by Day and Kelly (1969) and is treated in depth in Dubuc (1970).

In the case under consideration a description of enriched left Kan extensions has been given in terms of tensor products of functors by Cigler (1976), and shows thus that left Kan extensions exist. We are not in need, however, of introducing the notion of the tensor product of functors since the Kan extensions we are interested in may be given a simplified description.

Letting $\mathfrak{A}=\mathscr{F}^{\mathfrak{B}}$, we have the situation discussed in Herz and Pelletier (1976). There we gave the following description of $\operatorname{Lan}_{I}(F)(X)$ :

$$
\operatorname{Lan}_{I}(F)(X)=\lim _{\rightarrow}\{F(Y): Y \subset X, Y \in \mathfrak{F B}\} .
$$

It is easy to verify that this description as a colimit over finite dimensional subspaces of $X$ and inclusion maps actually gives us a left adjoint to $U$; since any two left adjoints of the same functor are naturally equivalent, this colimit is the left Kan extension.

We say that $F$ is $\mathscr{F}$-computable (previously called 'computable') when $\operatorname{Lan}_{I}(U F)=F$. A shorthand for $\operatorname{Lan}_{I}(U F)$ is $F^{L}$, the ' $\widetilde{F}$-computable part' of $F$.

The principal duality thoorem in Herz and Pelletier (1976) is the following:

1.1. THEOREM If $F$ is a F-computable functor, then $\psi_{X}^{*}: D F\left(X^{\prime}\right) \rightarrow(F X)^{\prime}$ is an isomorphism for every $X \in \mathfrak{B}$, where $\psi_{X}^{*}(\gamma)=$ tr $_{X} \circ \gamma_{X}$ and $\operatorname{tr}_{X}: X^{\prime} \otimes X \rightarrow \mathbf{R}$ denotes the trace map $\operatorname{tr}_{X}\left(\Sigma x_{i}^{\prime} \otimes x_{i}\right)=\Sigma x_{i}^{\prime}\left(x_{i}\right)$.

\section{The Radon-Nikodym Theorem}

We recall the computable functor $A^{\cdot-}$, which forms the basis of the applications given in Herz and Pelletier (1976) Section 3 to the duality Theorem 1.1. $A \cdot X$ is defined to be the closure of $\tau_{X}(A \otimes X)$ in $\operatorname{HOM}\left(A^{\prime}, X\right)$, where 
$\tau_{X}: A \otimes X \rightarrow H O M\left(A^{\prime}, X\right)$ is the $\mathfrak{B}$-natural transformation given by

$$
\tau_{\mathrm{X}}\left(\Sigma a_{i} \otimes x_{i}\right)\left(a^{\prime}\right)=\Sigma a^{\prime}\left(a_{i}\right) x_{i},
$$

for $a^{\prime} \in A^{\prime}$. We point out that $A^{\prime} \cdot X$ is also the image of $A^{\prime} \otimes X$ in $\operatorname{HOM}(A, X)$ under the obvious map. It is shown in the above-mentioned work that $A^{\prime} \cdot X=\operatorname{HOM}^{L}(A, X)$ and that if $A$ satisfies the approximation condition, then $\operatorname{HOM}^{L}(A, X)=\operatorname{COMP}(A, X)$, the compact operators from $A$ to $X$.

One special case of the functor $A \cdot-$ occurs when $A=C(\Omega)$, where $\Omega$ is a compact Hausdorff space. Then if $C(\Omega,-)$ denotes the functor sending $X \in \mathfrak{B}$ to the space of all continuous real-valued functions from $\Omega$ to $X$, we have $C(\Omega, X)=C(\Omega) \cdot X$, where $C(\Omega)=C(\Omega, \mathbf{R})$.

It evolves in Section 3 of Herz and Pelletier (1976) that $D(A \cdot-)(X)=\operatorname{INT}(A, X)$, the integral operators in the sense of Grothendieck from $A$ to $X$. Accordingly, from 1.1 it follows that the map

$$
\psi_{X}^{*}: I N T\left(A, X^{\prime}\right) \rightarrow(A \cdot X)^{\prime}
$$

is an isometric isomorphism. We also note that $\operatorname{INT}(A, X)$ is functorial in both variables. Although the dual of a computable functor, $\operatorname{INT}(A,-)$ is not itself computable since $I N T(A, X)=A^{\prime} \otimes X$ for $X$ (or $A$ ) finite dimensional implies that $I N T^{L}(A, X)=A^{\prime} \otimes X$.

Among the usual results concerning integral operators (see Grothendieck (1966)) which fall out elegantly from our categorical definition are the following factorization theorem and its corollary:

2.1. TheOrem. $T \in I N T(A, X)$ if and only if $i_{X} \circ T$ can be factored as:

$$
A \rightarrow L_{\infty}(\mu) \stackrel{Q}{\longrightarrow} L_{1}(\mu) \rightarrow X^{\prime \prime},
$$

where $i_{X}: X \rightarrow X^{\prime \prime}$ is the canonical isometric inclusion, $(\mu)$ denotes a compact measure space, and $Q: L_{\infty}(\mu) \rightarrow L_{1}(\mu)$ is the canonical inclusion.

2.2. Corollary. $T \in I N T\left(A, X^{\prime}\right)$ if and only if $T$ can be factored as:

$$
A \rightarrow L_{\infty}(\mu) \stackrel{Q}{\longrightarrow} L_{1}(\mu) \rightarrow X^{\prime},
$$

for $(\mu)$ a compact measure space and $Q$ the canonical map.

We mention at this point that the notion of an operator being 1-integral (Persson and Pietsch (1969)) is beginning to receive more attention than that of being integral in the above sense because of its connection to the Radon-Nikodym property as we see below and its generalizations to p-integrability. An operator 
$T: A \rightarrow X$ is said to be 1-integral when it factors as follows:

$$
A \rightarrow L_{\infty}(\mu) \stackrel{Q}{\longrightarrow} L_{1}(\mu) \rightarrow X .
$$

As a consequence of 2.2 , we see that $I N T_{1}\left(-, X^{\prime}\right)=I N T\left(-, X^{\prime}\right)$, for all $X \in \mathfrak{B}$, so that the two notions agree for dual spaces. Thus on dual spaces we may avail ourselves of the fact that $I N T_{1}\left(A, X^{\prime}\right)=D(A \cdot-)\left(X^{\prime}\right)$.

We let $T C(A, X)$ denote the Banach space of trace class operators (often called nuclear operators) from $A$ to $X$, that is $T C(A, X)$ consists of operators defined from elements of $A^{\prime} \otimes X$ as follows

$$
\left(\Sigma a_{i}^{\prime} \otimes x_{i}\right)(a)=\Sigma a_{i}^{\prime}(a) x_{i} \text { for } a \in A .
$$

The norm on $\operatorname{TC}(A, X)$ is the quotient norm it inherits from $A^{\prime} \otimes X$. We note that $T C(A, X)$ is functorial in both variables. We have $\mathfrak{B}$-bi-natural transformations $\theta_{A, X}, \pi_{A, X}, \varphi_{A, X}$ forming the following commutative triangle:

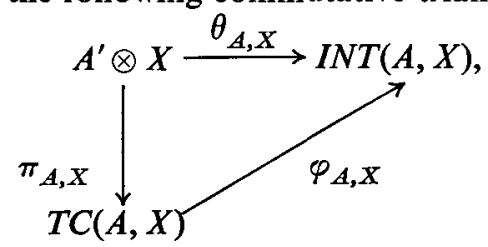

where $\theta_{A, X}\left(\Sigma a_{i}^{\prime} \otimes x_{i}\right)(a)=\Sigma a_{i}^{\prime}(a) x_{i}, \pi_{A, X}$ is the quotient map and $\varphi_{A, X}$ is the obvious morphism.

We say that $X^{\prime}$ satisfies the Radon-Nikodym property ('RN') if

$$
\operatorname{INT}\left(A, X^{\prime}\right)=T C\left(A, X^{\prime}\right)
$$

for all $A \in \mathfrak{B}$. $A$ is said to satisfy the dual Radon-Nikodym property ('RN*') if $\operatorname{INT}(A, X)=T C(A, X)$ for all $X \in \mathfrak{B}$.

The connection between ' $\mathrm{RN}$ ' and ' $\mathrm{RN}^{*}$ ' is given in the next proposition, but as with so many results in $\mathfrak{B}$, a hypothesis involving the approximation property is present. (We say that $X$ satisfies the approximation property ('A') whenever $\pi_{A, X}: A^{\prime} \otimes X \rightarrow T C(A, X)$ is an isomorphism for all $A \in \mathfrak{B}$.)

2.3. Proposition If $X$ satisfies ' $R N^{*}$ ', then $X$ ' satisfies ' $R N$ '. If $X$ ', satisfies ' $R N$ ' and ' $A$ ', then $X$ satisfies ' $R N$ ''.

Proof. The proof of the first statement follows from the fact that

and

$$
I N T\left(A, X^{\prime}\right) \cong I N T\left(X, A^{\prime}\right)
$$

$$
T C\left(X, A^{\prime}\right) \cong T C\left(A, X^{\prime}\right) .
$$

Let us assume that $X^{\prime}$ satisfies 'RN'. Then we have $\operatorname{INT}(X, A) \subset T C\left(X, A^{\prime \prime}\right)$, 
since $\operatorname{INT}(X, A) \subset I N T\left(X, A^{\prime \prime}\right)$ (see Herz and Pelletier (1976), 2.9) and

$$
I N T\left(X, A^{\prime \prime}\right) \cong I N T\left(A^{\prime}, X^{\prime}\right) \cong T C\left(A^{\prime}, X^{\prime}\right) \cong T C\left(X, A^{\prime \prime}\right) .
$$

Hence, we need to show that if $i_{A} \circ T \in T C\left(X, A^{\prime \prime}\right)$ and $T \in I N T(X, A)$, then $T \in T C(X, A)$. However, $i_{A} \circ T$ corresponds to $T^{\prime}$ under $T C\left(X, A^{\prime \prime}\right) \cong T C\left(A^{\prime}, X^{\prime}\right)$. Finally, we make use of the standard fact (see Lotz (1971), p. 30) that if $X^{\prime}$ satisfies ' $A$ ', then $T^{\prime} \in T C\left(A^{\prime}, X^{\prime}\right)$ implies $T \in T C(X, A)$.

Our main result of this section cannot be claimed to be new, belonging as it does to the folklore on Radon-Nikodym theorems. (Diestel (1975), Diestel and Uhl (1976) and Grothendieck (1966) are sources for most of this folklore.) However, its proof relies largely on categorical means which in part eliminates and in whole simplifies the analysis involved in the usual presentations.

2.4. THEOREM. The following statements are equivalent:

(1) $X^{\prime}$ satisfies ' $R N$ '.

(2) The canonical map $v_{A, X}: A^{\prime} \otimes X^{\prime} \rightarrow(A \cdot X)^{\prime}$ given by

$$
v_{A, x}\left(\Sigma a_{i}^{\prime} \otimes x_{i}^{\prime}\right)\left(\Sigma a_{j} \cdot x_{j}\right)=\Sigma \Sigma a_{i}^{\prime}\left(a_{j}\right) x_{i}^{\prime}\left(x_{j}\right)
$$

is a quotient for all $A \in \mathfrak{B}$.

(3) The canonical map $R: L_{\infty}\left(\mu, X^{\prime}\right) \rightarrow H O M\left(L_{1}(\mu), X^{\prime}\right)$ is onto, where $(\mu)$ is a compact measure space.

(4) $\operatorname{INT}\left(C(\Omega), X^{\prime}\right)=C(\Omega)^{\prime} \otimes X^{\prime}$ for every compact Hausdorff space $\Omega$.

ProOF. (1) $\Leftrightarrow(2)$ is obvious since $v_{A, X}$ is the following composite map

$$
A^{\prime} \otimes X^{\prime} \underset{\pi_{A, X^{\prime}}}{\longrightarrow} T C\left(A, X^{\prime}\right) \underset{\varphi_{A, X^{\prime}}}{\longrightarrow} \operatorname{INT}\left(A, X^{\prime}\right) \underset{\psi_{A, X}^{*}}{\stackrel{\cong}{\longrightarrow}}(A \cdot X)^{\prime}
$$

(1) $\Rightarrow$ (3). Let $T \in H O M\left(L_{1}(\mu), X^{\prime}\right)$. Then $T \circ Q \in I N T\left(L_{\infty}(\mu), X^{\prime}\right)$ since $Q$ is integral (see Herz and Pelletier (1976), 4.9). By (1), we have

$$
T \circ Q \in L_{\infty}(\mu)^{\prime} \otimes X^{\prime} \cong T C\left(L_{\infty}(\mu), X^{\prime}\right)
$$

However, under the isomorphism $T C\left(L_{\infty}(\mu), X^{\prime}\right) \cong T C\left(X, L_{\infty}(\mu)^{\prime}\right), \quad Q^{\prime} \circ T^{\prime} \circ i_{x}$ corresponds to $T \circ Q$ and is thus defined by the same element of $L_{\infty}(\mu)^{\prime} \otimes X^{\prime}$. On the other hand, one can easily verify that $\mathrm{Q}^{\prime}=i_{L_{1}} \circ Q$ :

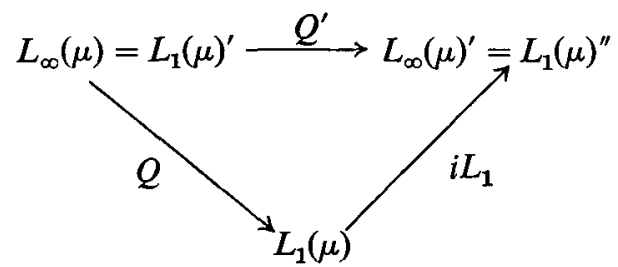


Hence, $\mathrm{Q}^{\prime} \circ T^{\prime} \circ i_{X}=i_{L_{1}} \circ Q \circ T^{\prime} \circ i_{X}$, and therefore, since $Q \circ T^{\prime} \circ i_{X} \in I N T\left(X, L_{1}(\mu)\right)$, $Q \circ T^{\prime} \circ i_{X} \in T C\left(X, L_{1}(\mu)\right)$ by the same argument given in the proof of 2.3. Thus, we see that $Q^{\prime} \circ T^{\prime} \circ i_{X}$ and, hence, $T \circ Q$ are defined by an element, say $f$, of $L_{1}(\mu) \otimes X^{\prime} \cong L_{1}\left(\mu, X^{\prime}\right)$. We have for every $g \in L_{\infty}(\mu), \quad T \circ Q(g)=T(g)=\int f g d \mu$. Clearly, then for every $g \in L_{1}(\mu)$, we have $T(g)=\int f g d \mu$, and by this property we see that $f$ must be essentially bounded, that is $f \in L_{\infty}\left(\mu, X^{\prime}\right)$.

(3) $\Rightarrow(4)$. Let $T \in I N T\left(C(\Omega), X^{\prime}\right)$. By the factorization theorem we have $T$ expressed as the composite

$$
C(\Omega) \rightarrow L_{\infty}(\mu) \underset{Q}{\longrightarrow} L_{1}(\mu) \underset{S}{\longrightarrow} X^{\prime} .
$$

By (3), there is an element $f \in L_{\infty}\left(\mu, X^{\prime}\right)$ such that for all $g \in L_{1}(\mu)$,

$$
S(g)=\int f g d \mu \text {. }
$$

Hence, for all $g \in C(\Omega)$, we have $T(g)=\int f g d \mu$. However,

$$
L_{\infty}\left(\mu, X^{\prime}\right) \subset L_{1}\left(\mu, X^{\prime}\right) \cong L_{1}(\mu) \otimes X^{\prime},
$$

and under this correspondence we have $f=\Sigma h_{i} \otimes x_{i}^{\prime}, h_{i} \in L_{1}(\mu), x_{i}^{\prime} \in X^{\prime}$. Clearly, we can view the $h_{i}$ as elements of $C(\Omega)^{\prime}$ and so $T \in C(\Omega)^{\prime} \otimes X^{\prime}$.

(4) $\Rightarrow(1)$. Let $A \in \mathfrak{B}$ and let $j: A \rightarrow C(\Omega)$ be an isometric inclusion. Consider the following diagram, which is commutative by the naturality of $\varphi$ :

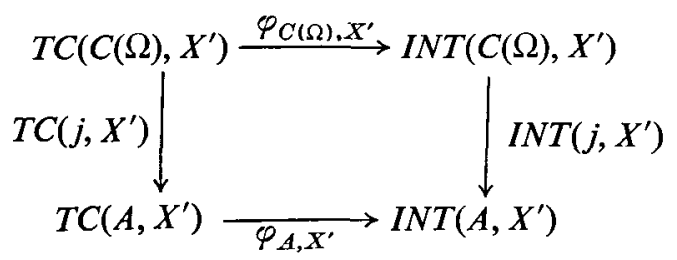

$\varphi_{C(\Omega), X^{\prime}}$ is a quotient map by (4), $I N T\left(j, X^{\prime}\right)$ is a quotient map since

$$
\operatorname{INT}\left(j, X^{\prime}\right)=(j \cdot X)^{\prime},
$$

and $T C\left(j, X^{\prime}\right)$ is obviously a quotient map. Therefore, $\varphi_{A, X}$, is also a quotient, and since it is a monomorphism as well, it is an isomorphism.

2.5. Corollary. If $X$ satisfies ' $R N^{*}$ ', then statements (2)-(4) of 2.4 hold.

2.6. Corollary. $X^{\prime}$ satisfies ' $R N$ ' and ' $A$ ' if and only if $A^{\prime} \otimes X^{\prime} \cong(A \cdot X)$ '.

The classical Dunford-Pettis theorem (Diestel and Uhl (1976), p. 4) is a straightforward consequence of the above theorem and adjointness. 
2.7. THEOREM. If $X^{\prime}$ is separable, then $X^{\prime}$ satisfies ' $R N$ '.

Proof. We use criterion (3) of 2.4. By adjointness, we have

$$
H O M\left(L_{1}(\mu), X^{\prime}\right) \cong\left(L_{1}(\mu) \otimes X\right)^{\prime} \cong L_{1}(\mu, X)^{\prime} \cong L_{\infty}\left(\mu, X^{\prime}\right) .
$$

\section{Weakly compact and compact operators}

The two principal results concerning the Radon-Nikodym property are the Dunford-Pettis theorem (2.7) and the Phillips theorem, which states that all reflexive spaces satisfy ' $R N$ '. The path from the Dunford-Pettis theorem to the Phillips theorem involved a deep study of weakly compact subsets of Banach spaces and weakly compact operators (Phillips (1943)). In fact, the key result due to Phillips is that the range of a weakly compact operator from $L_{1}(\mu)$ is separable and one can readily deduce the Phillips theorem from the Dunford-Pettis theorem using this fact and the obvious fact that all operators to a reflexive space are weakly compact. One means of arriving at the Phillips theorem with a minimum of analysis is to prove that the range of an operator from $L_{1}(\mu)$ to a reflexive space is separable, then use the Dunford-Pettis theorem and the factorization theorem (2.2). In this paper we will refrain from actually giving this proof since it is standard, but rather we give new ways of viewing the class of weakly compact operators and of characterizing ' $\mathrm{RN}$ '.

Let $W C(A, X)$ denote the Banach space of weakly compact operators from $A$ to $X$, that is operators $T: A \rightarrow X$ such that $T^{\prime \prime}\left[A^{\prime \prime}\right] \subset X$. Alternatively, $W C(A, X)$ can be described as the space of all operators such that the weak closure of the image of the closed unit ball in $A$ is compact in the weak topology on $X$. An operator from $A$ to $X$ is said to be compact if the closure of the image of the closed unit ball in $A$ is compact in the norm topology on $X$. We denote the set of all compact operators from $A$ to $X$ by $\operatorname{COMP}(A, X)$. Both $W C(A, X)$ and $\operatorname{COMP}(A, X)$ are functorial in both variables. It is easy to see that there is a natural inclusion

$$
j: \operatorname{COMP}(A, X) \rightarrow W C(A, X) .
$$

We mentioned in Section 2 that if $A$ satisfies 'A', then

$$
\operatorname{COMP}(A, X)=A^{\prime} \cdot X=\operatorname{HOM}^{L}(A, X),
$$

that is that $\operatorname{COMP}(A,-)$ is the left Kan extension of $\operatorname{HOM}(A,-)$ along $I: \mathfrak{F}^{\mathfrak{B}} \rightarrow \mathfrak{B}$. We proceed to give an analogous definition of $W C(A,-)$ as the left Kan extension of $\operatorname{HOM}(A,-)$ along $I: \mathfrak{R B} \rightarrow \mathfrak{B}$. (We hope no confusion will result from calling both inclusion functors $I$.)

From the results of Cigler (1976), referred to in Section 2, we know that the 
left Kan extension of $\operatorname{HOM}(A,-)$ along $I: \mathfrak{R B} \rightarrow \mathfrak{B}$ exists and can be expressed as a tensor product of functors. However, it is not hard to calculate directly that the left adjoint $L$ of $U$ is defined by

$$
L F X=\lim _{\rightarrow}\left\{F(Y):\left(T, f_{Y}\right) \in \mathfrak{D}(X)\right\},
$$

where $\mathfrak{D}(X)$ denotes the category of pairs $\left(Y, f_{Y}\right), Y \in \mathfrak{R B}$ and $f_{Y}: Y \rightarrow X$ a monomorphism, whose morphisms $h:\left(Y, f_{Y}\right) \rightarrow\left(Y^{\prime}, f_{Y^{\prime}}\right)$ are maps $h: Y \rightarrow Y^{\prime}$ such that $f_{Y}=f_{Y^{\prime}}$ oh. Hence, $L F X=\operatorname{Lan}_{I}(F)(X)$. For shorthand we will denote $\operatorname{Lan}_{I}(U F)$ for $F \in \mathfrak{B}^{\mathfrak{B}}$ by $F^{\boldsymbol{W}}$.

The proof that $\operatorname{HOM}^{W}(A,-)$ is $W C(A,-)$ is based on the recent important result of Davis et al. (1974) that every weakly compact operator in $\mathfrak{B}$ factors through a reflexive Banach space. To summarize briefly, they define for $T \in H O M(A, X)$ a norm on a subspace of $X$ using the convex symmetric bounded set $T\left(B_{A}\right)$, where $B_{A}$ is the closed unit ball of $A$, such that the resulting space, call it $X(T)$, is reflexive if and only if $T \in W C(A, X)$. Moreover, $T \in W C(A, X)$ can be factored as

$$
A \stackrel{\mathcal{T}}{\longrightarrow} X(T) \stackrel{R_{T}}{\longrightarrow} X,
$$

where $R_{T}$ is a continuous monomorphism in $\mathfrak{B}$, and $\widetilde{T}=R_{T}^{-1} \circ T \in H O M(A, X(T))$.

\subsection{THEOREM. $W C(A, X)=\operatorname{HOM}^{\boldsymbol{W}}(A, X)$.}

Proof. For any $\left(Y, f_{Y}\right) \in \mathfrak{D}(X)$, we have the map

$$
\operatorname{HOM}\left(A, f_{Y}\right): \operatorname{HOM}(A, Y) \rightarrow W C(A, X),
$$

and the family $\left\{\operatorname{HOM}\left(A, f_{Y}\right)\right\}$ is compatible with maps in $\mathfrak{D}(X)$. Let

$$
Z=\lim _{\vec{D}(X)} \operatorname{HOM}(A, Y),
$$

equipped with the family of injections $g_{Y}: \operatorname{HOM}(A, Y) \rightarrow Z$. Then there exists a unique map $\varphi: Z \rightarrow W C(A, X)$ such that $\varphi \circ g_{Y}=\operatorname{HOM}\left(A, f_{Y}\right)$ for all $\left(Y, f_{Y}\right) \in \mathfrak{D}(X)$. We define $\psi: W C(A, X) \rightarrow Z$ as follows: let $T \in W C(A, X)$ be factored as

$$
\stackrel{\tilde{T}}{\longrightarrow} X(T) \stackrel{R_{T}}{\longrightarrow} X
$$

as described above. Since $\left(X(T), R_{T}\right) \in \mathfrak{D}(X)$, we may define $\psi(T)=g_{X(T)}(T)$. We claim that $\psi$ is such that $\psi \operatorname{HOM}\left(A, f_{Y}\right)=g_{Y}$ for all $\left(Y, f_{Y}\right) \in \mathcal{D}(X)$ and that $\psi$ and $\varphi$ are inverses. The latter result will show that $\psi \in \mathfrak{B}(W C(A, X), Z)$.

Let $\left(Y, f_{Y}\right) \in \mathfrak{D}(X), T \in H O M(A, Y)$. Consider the following diagram: 


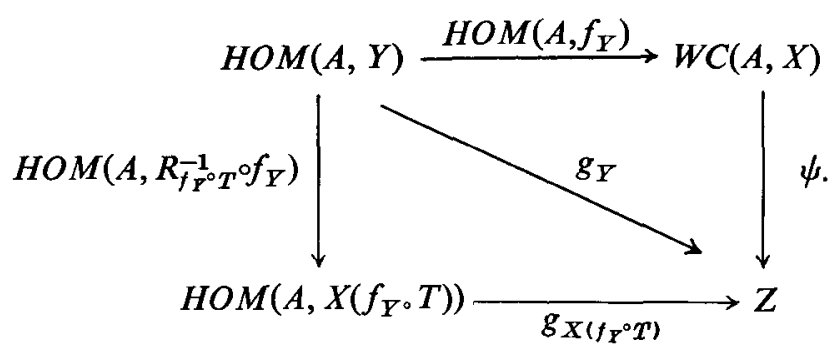

We have

$$
\begin{aligned}
\left(\psi \circ H O M\left(A, f_{Y}\right)\right)(T) & =\psi\left(f_{Y} \circ T\right)=g_{X\left(f_{Y} \circ T\right)}\left(\widetilde{f_{Y} \circ T}\right) \\
& =\left(g_{X_{\left(f_{Y} \circ T\right)} \circ H O M\left(A, R_{f_{Y} \circ T}^{-1} \circ f_{Y}\right)}\right)(T)=g_{Y}(T)
\end{aligned}
$$

Hence, $\psi \circ H O M\left(A, f_{Y}\right)=g$ for all $\left(Y, f_{Y}\right) \in \mathfrak{D}(X)$.

Since $\varphi \circ g_{Y}=\operatorname{HOM}\left(A, f_{Y}\right)$, we have $\psi \circ \varphi \circ g_{Y}=g_{Y}$ for all $\left(Y, f_{Y}\right) \in \mathcal{D}(X)$ and, therefore, by definition of $Z, \psi \circ \varphi=1_{z}$. However, $\varphi$ is surjective since for $T \in W C(A, X)$,

$$
\varphi \circ g_{X(T)}(\tilde{T})=T
$$

Hence, $\varphi \circ \psi \circ \varphi=\varphi$ implies that $\varphi \circ \psi=1_{W C(A, x)}$ and $\varphi$ and $\psi$ are inverses as claimed.

We remark here that the above interesting characterizations of $W C(A,-)$ and of $\operatorname{COMP}(A,-)$ for $A$ satisfying ' $\mathrm{A}$ ' as $\mathrm{Kan}$ extensions will most likely lead to further general results of this type which we intend to pursue at a later date.

\section{Dual functors and the Radon-Nikodym property}

In this final section we study the meaning of the Radon-Nikodym property from a point of view hitherto not taken in the literature. The constructions of $F^{L}$ and $F^{W}$ play a large role in our results, revealing the central nature of these limits. We find that there are interesting relationships between the Radon-Nikodym property and the agreement of certain pairs of dual functors, namely the duals of $\operatorname{COMP}(A,-)$ and $W C(A,-)$ or the duals of $\operatorname{INT}(A,-)$ and $I N T^{L}(A,-)$. Usually, we shall require the assumption of ' $\mathrm{A}$ ' on certain of the spaces in question.

The first result we shall give is a new statement of the definition of 'RN', the proof of which is trivial (assuming the Phillips theorem).

4.1. TheOREM. $X^{\prime}$ satisfies ' $R N$ ' if and only if $I N T\left(A, X^{\prime}\right)=I N T^{W}\left(A, X^{\prime}\right)$ for all $A \in \mathfrak{B}$ such that $A$ ' satisfies ' $A$ '. 
Proof. INT $T^{\mathscr{W}}\left(A, X^{\prime}\right)=\underset{\mathfrak{D}\left(X^{\prime}\right)}{\underset{\lim }{\longrightarrow}} \operatorname{INT}(A, Y)=\underset{\mathfrak{D}\left(X^{\prime}\right)}{\lim } T C(A, Y) \cong \underset{\underset{\mathfrak{D}\left(X^{\prime}\right)}{\longrightarrow}}{\lim } T C\left(Y^{\prime}, A^{\prime}\right)$

$$
\begin{aligned}
& \cong \underset{\mathfrak{D}\left(X^{\prime}\right)}{\lim } Y \otimes A^{\prime}=X^{\prime} \otimes A^{\prime} \cong T C\left(X, A^{\prime}\right) \\
& \cong T C\left(A, X^{\prime}\right) .
\end{aligned}
$$

The key to many of the following results on dual functors is the tensor contraction map

$$
\psi_{X, Y}: D F X \otimes F Y \rightarrow X \otimes Y
$$

defined by $\psi_{X, Y}(\gamma \otimes y)=\gamma_{Y}(y)$, which is discussed in detail in Herz and Pelletier (1976). For example, one case we consider, for $A$ satisfying ' $\mathrm{A}$ ', is $F=A^{\prime} \cdot-=\operatorname{COMP}(A,-)$ and $D F=I N T\left(A^{\prime},-\right)$. It will be worthwhile to examine the behaviour of $\psi_{X, Y}$ in detail in this paradigm case before we proceed.

\subsection{LEMMA. The map}

$$
\psi_{X, Y}: \operatorname{INT}\left(A^{\prime}, X\right) \otimes C O M P(A, Y) \rightarrow X \otimes Y
$$

behaves as follows:

$$
\psi_{X, Y}(T \otimes f)=T \circ f^{\prime},
$$

when $\psi_{X, Y}(T \otimes f)$ is viewed as a map from $Y^{\prime}$ to $X$.

Proof. Under the isomorphism $\operatorname{DCOMP}(A,-)(X)=\operatorname{INT}\left(A^{\prime}, X\right), \gamma$ maps to $\gamma_{\mathrm{R}}$, that is $T=\gamma_{\mathrm{R}}$ for some $\gamma \in D \operatorname{COMP}(A,-)(X)$. Since $\psi_{X, Y}(\gamma \otimes f)=\gamma_{\mathrm{Y}}(f)$, we must, therefore, show that for every $y^{\prime} \in Y^{\prime}$,

$$
\left(X \otimes y^{\prime}\right)\left(\gamma_{Y}(f)\right)=\left(\gamma_{\mathbf{R}} \circ f^{\prime}\right)\left(y^{\prime}\right) .
$$

However, this equality is a consequence of the commutativity of the following diagram, which is due to the naturality of $\gamma$ :

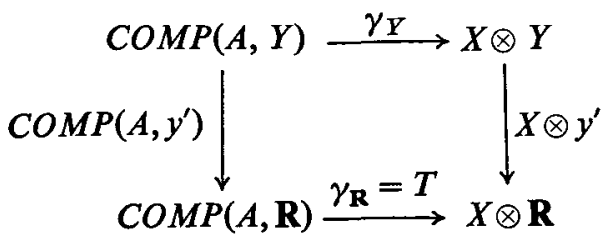

Making use of the tensor contraction map, we see in the next result that the agreement of $D I N T(A,-)$ and $D\left(I N T^{L}\right)(A,-)$ is connected to ' $R N^{\prime}$ ' in the presence of ' $A$ '. We remark that Losert has independently proved the same result on the 
category of Banach spaces satisfying the metric approximation property in his doctoral dissertation (Losert (1976)) but that his result does not follow from a purely categorical method of proof as ours does.

4.3. Theorem. Let $A$ ' satisfy ' $A$ '. Then $A$ ' satisfies ' $R N$ ' if and only if

$$
\operatorname{DINT}(A,-)=D\left(I N T^{L}\right)(A,-) .
$$

Proof. If $A$ ' satisfies ' $\mathrm{RN}$ ', then by $2.3, A$ satisfies ' $\mathrm{RN}$ '' and so we have $\operatorname{INT}(A,-)=T C(A,-)$. We claim that $T C(A,-)=A^{\prime} \otimes-$. Since $A^{\prime}$ satisfies ' $\mathrm{A}$ ', we have

$$
T C\left(A, X^{\prime}\right) \cong T C\left(X, A^{\prime}\right) \cong X^{\prime} \otimes A^{\prime}
$$

for all $X \in \mathfrak{B}$. However, the following diagram is commutative by the naturality of $\pi_{A,-}$ :

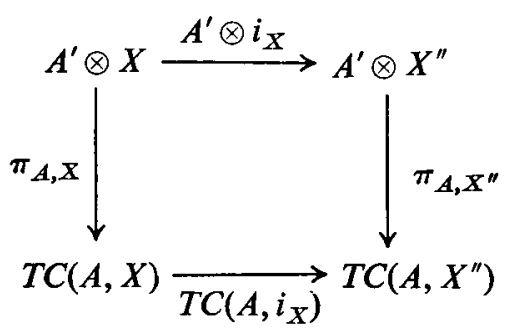

Since $\pi_{A, X^{\prime \prime}}$ is an isomorphism and $A^{\prime} \otimes i_{X}$ is an isometric inclusion, the composition $\operatorname{TC}\left(A, i_{X}\right) \circ \pi_{A, X}$ is an isometric inclusion. Hence, $\pi_{A, X}$ is an isometry and a quotient map, which implies that it is an isomorphism. Thus, our claim is proved, and we have $\operatorname{INT}(A,-)=A^{\prime} \otimes-=I N T^{L}(A,-)$ and so

$$
\operatorname{DINT}(A,-)=\operatorname{DINT}^{L}(A,-) \text {. }
$$

On the other hand, suppose that $\operatorname{DINT}(A,-)=\operatorname{DINT}^{L}(A,-)=\operatorname{HOM}\left(A^{\prime},-\right)$. Then we may apply the tensor contraction map as follows:

$$
\psi_{A^{\prime}, X}: \operatorname{DINT}(A,-) A^{\prime} \otimes I N T(A, X) \rightarrow A^{\prime} \otimes X .
$$

Moreover, using a proof identical to that of the above lemma, we may see that

$$
\psi_{A^{\prime}, X}(f \otimes T)=f \circ T^{\prime}
$$

for $f \in H O M\left(A^{\prime}, A^{\prime}\right)$ and $T \in I N T(A, X)$. Hence

$$
\psi_{A^{\prime}, X}\left(1_{A^{\prime}} \otimes T\right)=T^{\prime} \in A^{\prime} \otimes X=T C(A, X)
$$

for any $T \in I N T(A, X)$. Hence, we have $T \in T C(A, X)$ and so $A$ satisfies 'RN*' and $A$ ' satisfies 'RN'.

A corollary of the Phillips theorem emphasizes the relationship between the duals of the compact and weakly compact operators and ' $R N$ '. 
4.4. Corollary. If $A$ is reflexive and satisfies ' $A$ ', then

$$
\operatorname{DCOMP}(A,-)=D W C(A,-) \text {. }
$$

Proof. We have

$$
\operatorname{DCOMP}(A,-)=\operatorname{INT}\left(A^{\prime},-\right)
$$

and

$$
D W C(A,-)=\operatorname{DHOM}(A,-)=A \otimes-\cong A^{\prime \prime} \otimes-.
$$

Hence, $\operatorname{DCOMP}(A,-)=D W C(A,-)$ if and only if $A^{\prime}$ 'satisfies ' $\mathrm{RN}$ '' if and only if (in the presence of ' $\mathrm{A}$ ') $A \cong A^{\prime \prime}$ satisfies ' $\mathrm{RN}$ '.

We remark from the above proof that the statement

$$
\operatorname{DCOMP}(A,-)=D W C(A,-)
$$

is another characterization of 'RN' for reflexive spaces $A$.

For general spaces 'RN' continues to imply agreement of the duals of the compact and weakly compact operators, but more assumption of ' $A$ ' is required.

\subsection{TheOREM. Let $X^{\prime}$ and $A$ satisfy ' $A$ '. If INT $\left(A^{\prime}, X^{\prime}\right)=T C\left(A^{\prime}, X^{\prime}\right)$, then}

$$
\operatorname{DCOMP}(A,-) X^{\prime}=D W C(A,-) X^{\prime} .
$$

Proof. We define a map

$$
\tau: I N T\left(A^{\prime}, X^{\prime}\right) \rightarrow D W C(A,-) X^{\prime}
$$

by $\tau(T)_{Y}(f)=T \circ f^{\prime}$ for $f \in W C(A, Y)$. Since $T \in T C\left(A^{\prime}, X^{\prime}\right)$ by hypothesis,

$$
T \circ f^{\prime} \in T C\left(Y^{\prime}, X^{\prime}\right) \text {. }
$$

However, $T C\left(Y^{\prime}, X\right) \cong T C\left(X, Y^{\prime \prime}\right)$, and under this isomorphism $T$ of corresponds to $f^{\prime \prime} \circ T^{\prime} \circ i_{X}$. Moreover, since $f$ is weakly compact, the image of $f^{\prime \prime} \circ T^{\prime} \circ i_{X}$ lies in $Y$ and so $T \circ f^{\prime} \in T C(X, Y) \cong X^{\prime} \otimes Y$, and $\tau$ is well defined.

We claim that $\tau$ and $\sigma=D(j)_{X^{\prime}}$, where $j: \operatorname{COMP}(A,-) \rightarrow W C(A,-)$ is the inclusion mentioned in Section 3, are inverse to each other. (Recall that

$$
\operatorname{DCOMP}(A,-) X^{\prime}=D\left(A^{\prime} \cdot\right) X^{\prime}=I N T\left(A^{\prime}, X^{\prime}\right)
$$

when $A$ satisfies 'A' via $v \rightarrow v_{\mathbf{R}}$.) To wit, let $T \in I N T\left(A^{\prime}, X^{\prime}\right)$. Then

$$
(\sigma \circ \tau)(T)=\tau(T)_{\mathbf{R}} \in D C O M P(A,-) X^{\prime}
$$

and if $f \in \operatorname{COMP}(A, \mathbf{R})=A^{\prime}$, then $f^{\prime}: \mathbf{R} \rightarrow A^{\prime}$ is equal to $\hat{f}$, where $\hat{f}(1)=f$. Thus, we have

$$
\tau(T)_{\mathbf{R}}(f)=T \circ \hat{f}=T(f)
$$

and so $(\sigma \circ \tau)(T)=T$. 
Now let $\gamma \in D W C(A,-) X^{\prime}$. Then $(\tau \circ \sigma)(\gamma)=\tau\left(\gamma_{\mathrm{R}}\right) \in D W C(A,-) X^{\prime}$. So if $f \in W C(A, Y)$, then

$$
(\tau \circ \sigma)(\gamma)_{Y}(f)=\tau\left(\gamma_{R}\right)(f)=\gamma_{R} \circ f^{\prime} .
$$

To show that $\gamma_{R}$ of acts like $\gamma_{Y}(f)$ as a map from $Y^{\prime}$ to $X^{\prime}$, we follow the same proof as given in Lemma 4.2, using the naturality of $\gamma$. Hence,

$$
(\tau \circ \sigma)(\gamma)_{Y}(f)=\gamma_{Y}(f)
$$

and so $(\tau \circ \sigma) \gamma=\gamma$.

4.6. Corollary. If $A$ ' satisfies ' $R N^{*}$ ' and ' $A$ ', then

$$
\operatorname{DCOMP}(A,-) X^{\prime}=\operatorname{DWC}(A,-) X^{\prime}
$$

for every $X$ ' satisfying ' $A$ '.

Moving in the opposite direction of this result, we prove under the assumption of $\operatorname{DCOMP}\left(A^{\prime},-\right)=D W C\left(A^{\prime},-\right)$ and ' $\mathrm{A}$ ' that there is a natural isometric inclusion of $W C\left(A^{\prime},-\right)$ in $\operatorname{DINT}(A,-)(-)$. We note that this result can be proved without the above assumption using the strong result (a key to the classical RadonNikodym theorems) that an integral operator composed with a weakly compact operator yields a trace class operator (see Grothendieck (1966), Section 4); J. Cigler claims to have an unpublished proof of this fact, and Losert (1976) includes such a result. However, we emphasize that we prefer our assumption on the dual functors in that the result is then obtainable by our categorical methods.

4.7. Theorem. Let $A^{\prime}$ satisfy ' $A$ '. If $\operatorname{DCOMP}\left(A^{\prime},-\right) X=D W C\left(A^{\prime},-\right) X$, then there exists an isometric inclusion

$$
\xi_{A, X}: W C\left(A^{\prime}, X\right) \rightarrow D I N T(A,-) X,
$$

which is natural in $X$.

ProOF We define $\xi_{A, X}$ as follows: for $f \in W C\left(A^{\prime}, X\right), \quad T \in I N T(A, Y)$, $\xi_{A, X}(f)_{Y}(T)=f \circ T^{\prime}$.

We must show that $f \circ T^{\prime} \in X \otimes Y$, and, hence, that $\xi_{A, X}(f) \in D I N T(A,-) X$. (Verifying that $\xi$ is natural is easy.) By virtue of our hypothesis, we may consider the tensor contraction map

$$
\psi_{Y^{\prime \prime}, X}: I N T\left(A^{\prime \prime}, Y^{\prime \prime}\right) \otimes W C\left(A^{\prime}, X\right) \rightarrow Y^{\prime \prime} \otimes X ;
$$

thus, we have $\psi_{Y ", x}\left(T^{\prime \prime} \otimes f\right)=T^{\prime \prime} \circ f^{\prime} \in Y^{\prime \prime} \otimes X$. However, it is a standard fact that integral operators are weakly compact and so $T \in W C(A, Y)$ implies that $T^{\prime \prime} \circ f^{\prime} \in Y \otimes X$. Clearly then, $f \circ T^{\prime} \in X \otimes Y$, which proves that our definition of $\xi$ makes sense. 
To see that $\xi_{A, X}$ is an isometry, let $f \in W C\left(A^{\prime}, X\right)$ be non-zero. We have

$$
\begin{aligned}
\left\|_{A, X}(f)\right\| & =\sup \left\{\left\|\xi_{A, X}(f)_{Y}(T)\right\|: Y \in \mathfrak{B}, T \in I N T(A, Y),\|T\| \leqslant 1\right\} \\
& =\sup \left\{\left\|f \circ T^{\prime}\right\|: Y \in \mathfrak{B}, T \in I N T(A, Y),\|T\| \leqslant 1\right\} \leqslant\|f\| .
\end{aligned}
$$

However, $\|f\|=\sup \left\{\|f(\alpha)\|: \alpha \in A^{\prime},\|\alpha\| \leqslant 1\right\}$, and each $\alpha \in A^{\prime}$ may be regarded as an element of $\operatorname{INT}(A, \mathbf{R})$. Hence

$$
\begin{aligned}
\|f\| & =\sup \left\{\|f(\alpha)\|: \alpha \in A^{\prime},\|\alpha\| \leqslant 1\right\} \\
& =\sup \left\{\left\|f \circ \alpha^{\prime}\right\|: \alpha \in I N T(A, \mathbf{R}),\|\alpha\| \leqslant 1\right\} \\
& =\left\|\xi_{A, X}(f)_{\mathbf{R}}\right\| \leqslant\left\|\xi_{A, X}(f)\right\|,
\end{aligned}
$$

and so $\xi_{A, X}$ is an isometry.

For our final result we summarize the situation when $A$ is reflexive.

4.8. COROLlary. Let $A$ be reftexive and satisfy $A^{\prime}$. Then the following statements are equivalent:

(1) $\operatorname{DCOMP}(A,-)=\operatorname{DWC}(A,-)$.

(2) A satisfies ' $R N$ '.

(3) $\xi_{A^{\prime},-}: W C(A,) \rightarrow D I N T\left(A^{\prime},-\right)()$ exists and is a natural isometric inclusion.

Proof 4.4 and 4.7 give us $1 \Leftrightarrow 2$ and $1 \Rightarrow 3$.

On the other hand, the existence of $\xi_{A^{\prime}, X}$ means that $f \circ T^{\prime} \in X \otimes Y$ for all $f \in W C(A, X), T \in I N T\left(A^{\prime}, Y\right)$. Let $f=1_{A} \in W C(A, A)$. Then for all $T \in I N T\left(A^{\prime}, Y\right)$, $T^{\prime} \in A \otimes Y$. Hence, $T \in A \otimes Y \cong T C\left(A^{\prime}, Y\right)$, and so $I N T\left(A^{\prime}, Y\right) \cong T C\left(A^{\prime}, Y\right)$ for all $Y \in \mathfrak{B}$. Therefore, $A$ ' satisfies 'RN*' and $A$ satisfies 'RN'.

\section{Acknowledgements}

The author gratefully acknowledges the many helpful comments of $\mathrm{C}$. Herz received in conversations and correspondence. She also expresses her appreciation for the suggestions of F. E. J. Linton made on the first draft of the article and the recent insightful suggestions of the referee. During the preparation of this paper the author received partial support from the National Research Council of Canada Grant No. A9134.

\section{References}

J. Cigler (1976), Tensor products of functors on categories of Banach spaces (Springer Lecture Notes 540, 164-187).

W. J. Davis, T. Figiel, W. B. Johnson and A. Pelczynski (1974), 'Factoring weakly compact operators', J. Functional Analysis 17, 311-327. 
B. J. Day and G. M. Kelly (1969) Enriched functor categories (Springer Lecture Notes 106, 178-191.)

J. Diestel (1972), 'The Radon-Nikodym property and the coincidence of integral and nuclear operators', Rev. Roumaine Math. Pures Appl. 17, 1611-1620.

J. Diestel (1975), Geometry of Banach spaces-selected topics (Springer Lecture Notes 485).

J. Diestel and J. J. Uhl, Jr. (1976), 'The Radon-Nikodym Theorem for Banach space valued measures', Rocky Mountain J. Math. 6, 1-46.

E. J. Dubuc (1970), Kan extensions in enriched category theory (Springer Lecture Notes 145).

A. Grothendieck (1966), Produits tensoriels topologiques et espaces nucléaires (Mem. Amer. Math. Soc. 16).

C. Herz and J. Wick Pelletier (1976), 'Dual functors and integral operators in the category of Banach spaces', J. Pure Appl. Algebra 8, 5-22.

V. Losert (1976). Dualität von Funktoren und Operatorenideale (Dissertation, Universität Wien).

F. E. J. Linton (1965), 'Autonomous categories and duality of functors', J. Algebra 2, 315-349.

H. Lotz (1971), Topological tensor products, linear mappings and nuclear spaces (Lecture Notes, University of Illinois).

S. MacLane (1971), Categories for the working mathematician (Springer-Verlag, New York).

B. S. Mityagin and A. S. Švarc (1964), 'Functors in categories of Banach spaces', Russian Math. Surveys 19(2), 65-127.

A. Persson and A. Pietsch (1969), 'p-Nukleare und p-integrale Abbildungen', Studia Math. 33, $19-62$.

R. S. Phillips (1943), 'On weakly compact subsets of a Banach space', Amer. J. Math. 65, 108-136.

Department of Mathematics

York University, Keele Street

Downsview, Ontario

Canada 